\title{
Roof- and Pyramid Wavefront Sensing with Extended Sources
}

\author{
Brent Ellerbroek ${ }^{\mathrm{a}}$, Jean-Pierre Veran $^{\mathrm{b}}$, and Lianqi Wang ${ }^{\mathrm{c}}$ \\ ${ }^{a}$ Retired, Long Beach, USA \\ ${ }^{\mathrm{b}}$ NRC Herberg Institute of Astrophysics, Victoria, Canada \\ 'Thirty Meter Telescope International Observatory, Pasadena, USA
}

\begin{abstract}
We generalize the standard Fourier optics measurement equations for the roof- and pyramid wavefront sensor (RWFS and PWFS) concepts to the case of extended (non-point) sources. Samples of such sources include (i) extended astronomical objects such as moons, (ii) point sources with modulation (i.e., tip/tilt nutation), and (iii) elongated laser guide stars. For simple source profiles, for example top-hat or Gaussian functions, the effect of the extended source on the measurement can be represented analytically as a filter function for each of these sample cases. For the RWFS, we use this representation to evaluate the impact of extended source on the wavefront reconstruction error due to noise, as a function of the number of Zernike modes estimated.

A limited set of sample results are also presented for more computationally intensive case of the PWFS. The trends are qualitatively similar to the RWFS, but with a modestly larger sensitivity to noise when reconstructing 50 or more Zernike modes.

Finally, the measurement equations developed for extended sources indicated that the RWFS and PWFS wavefront measurements may be biased (i.e., nonzero measurements for a null wavefront) when the source is asymmetrical, but the magnitude of this effect has not been studied yet either analytically or via simulation.
\end{abstract}

Keywords: Pyramid wavefront sensing, extended source wavefront sensing

\section{INTRODUCTION}

Intuitively, the sensitivity of the pyramid wavefront sensor ${ }^{1}$ (PWFS) is reduced if measurements are made using a blurred or extended object as the guide star, instead of a point source. The purpose of this study is to model this effect analytically, and assess the resulting increase in wavefront reconstruction error due to measurement noise. We also consider the case of the roof WFS ${ }^{2}$ (RWFS). The two-channel RWFS provides a pair of bi-cell wavefront gradient measurements at each point in the pupil, instead of the quad cell gradient measurement obtained with the PWFS.
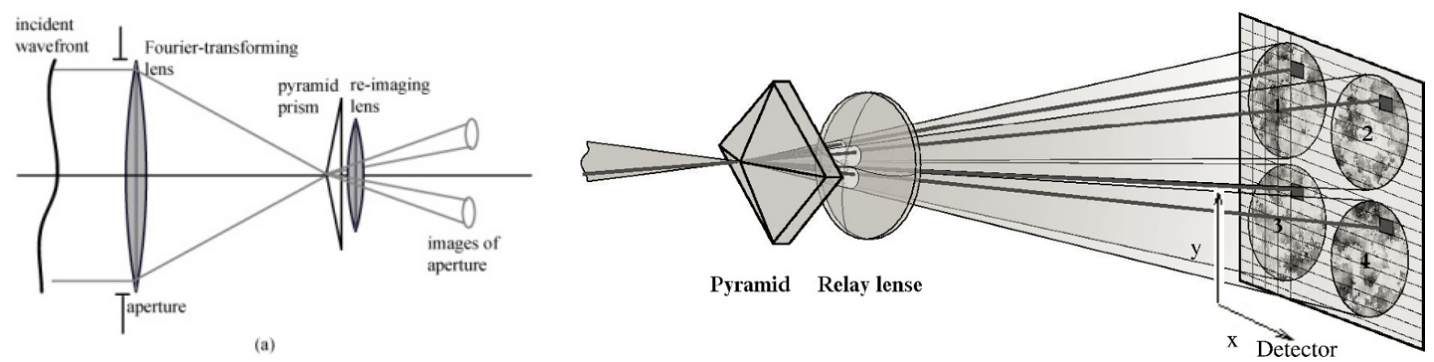

Figure 1. Basic illustrations of the roof- (left) and pyramid (right) wavefront sensing concepts.

Further author information: (Send correspondence to Brent Ellerbroek)

B.E: E-mail: ellerbroekbrent@gmail.com

L.W.: E-mail: lianqiw@tmt.org

J-P.V.: E-mail: jean-pierre.veran@nrc-cnrc.gc.ca 
Section 2 of this paper summarizes the RWFS and PWFS measurement equations developed for the extended source case. Section 3 plots sample Zernike mode influence functions computed using these formulas, to illustrate the similarity between the RWFS and PWFS results and the reduction in sensitivity caused by extended sources. Section 4 presents the sensitivity to noise of least squares wavefront reconstruction using the RWFS as a function of the number of modes estimated, and the dimension of the extended source. Section 5 presents similar sample results for the PWFS. Section 6 is a brief summary. Finally, the two appendices provide further detail on the derivation of the RWFS and PWFS measurement models.

\section{RWFS AND PWFS MEASUREMENT MODELS WITH EXTENDED SOURCES}

The standard Fourier optics model for the gradient measurement $g$ obtained with either the RWFS or PWFS and a point source guide star is given by:

$$
\begin{aligned}
g_{\{x, y\}}(\vec{r}, \phi) & =\sum_{i} w_{\{x, y\}, i} I\left(\vec{r}, \phi, m_{i}\right) \\
I(\vec{r}, \phi, m) & =\left|\int d \vec{\kappa} m(\vec{\kappa}) \exp (k \vec{r} \cdot \vec{\kappa}) \int d \vec{s} A(\vec{s}) \exp [i \phi(\vec{s})] \exp (-k \vec{s} \cdot \vec{\kappa})\right|^{2} .
\end{aligned}
$$

Here $\vec{r}$ is a point in the pupil, $\phi$ is the wavefront, the sum is taken over the 2 or 4 detector pixels used in the measurement, $w_{\{x, y\}, i}$ is the quad-cell (or bi-cell) weight for detector $i, m_{i}$ is the spatial filtering provided by the pyramid (or roof) for this detector, and $A(\vec{s})$ is the pupil function. From this starting point, Appendix A derives the standard measurement equations

$$
g_{x}(\vec{r}, \phi)=\lambda^{4} \int d t_{x} A(\vec{r}) A\left(r+\left(t_{x}, 0\right)\right)\left[\frac{\sin \left(\phi(\vec{r})-\phi\left(\vec{r}+\left(t_{x}, 0\right)\right)\right)}{\pi t_{x}}\right]
$$

for the RWFS, ${ }^{3}$ and

$$
\begin{aligned}
g_{x}(\vec{r}, \phi)= & \frac{\lambda^{4}}{2} \int d t_{x} A(\vec{r}) A\left(r+\left(t_{x}, 0\right)\right)\left[\frac{\sin \left(\phi(\vec{r})-\phi\left(\vec{r}+\left(t_{x}, 0\right)\right)\right)}{\pi t_{x}}\right] \\
& -\frac{\lambda^{4}}{2} \iint \frac{d t_{y}}{\pi t_{y}} \frac{d t_{y}^{\prime}}{\pi t_{y}^{\prime}} \int d t_{x} A\left(\vec{r}+\left(0, t_{y}^{\prime}\right)\right) A(\vec{r}+\vec{t})\left[\frac{\sin \left(\phi\left(\vec{r}+\left(0, t_{y}^{\prime}\right)\right)-\phi(\vec{r}+\vec{t})\right)}{\pi t_{x}}\right]
\end{aligned}
$$

for the PWFS.

For an extended and/or blurred guide star, we model the guide star as a superposition of discrete, incoherent point sources, with the wavefront $\phi$ adjusted by the appropriate tilt and/or focus terms at each point:

$$
g_{\{x, y\}}(\vec{r}, \phi)=\sum_{i} w_{\{x, y\}, i} \int d \vec{\theta} W(\vec{\theta}) \int d f p(f) I\left(\vec{r}, \phi+\phi_{o}, m_{i}\right) .
$$

Here $W$ is the intensity profile of an extended object (or the time-averaged profile of a modulated point source), $p$ is the vertical profile of an elongated guide star, and $\phi_{o}$ is the tip/tilt/focus adjustment made to the wavefront at each point in the extended object:

$$
\phi_{o}(\vec{r})=\phi_{o}(\vec{r} ; \vec{\theta}, f)=\frac{2 \pi \vec{\theta}}{\lambda / D} \cdot \frac{\vec{r}}{D}+2 \pi f\left|\frac{2 \vec{r}}{D}\right|^{2}
$$

Here $D$ is the aperture diameter, and the range variable $f$ is given in units of peak-to-valley waves of defocus across the aperture. 


\subsection{RWFS Case}

To develop an RWFS measurement formula for extended sources, we first commute the order of summation and integration in Eq. (5) above, then substitute Eq. (3) for the point source case with the wavefront $\phi+\phi_{o}$, and then commute the order of integration once again. The result is the expression

$g_{x}(\vec{r}, \phi)=\lambda^{4} \int d t_{x} A(\vec{r}) A\left(r+\left(t_{x}, 0\right)\right) \int d \vec{\theta} W(\vec{\theta}) \int d f p(f)\left[\frac{\sin \left(\phi(\vec{r})-\phi\left(\vec{r}+\left(t_{x}, 0\right)\right)+\phi_{o}(\vec{r})-\phi_{o}\left(\vec{r}+\left(t_{x}, 0\right)\right)\right)}{\pi t_{x}}\right]$.

Appendix B below describes how the two integrals with respect to $\vec{\theta}$ and $f$ can be evaluated for several sample cases. The first case is an idealized LGS with a top-hat elongation profile $p$ and a point source intensity profile $W$. The measurement formula then takes the form

$$
g_{x}(\vec{r}, \phi)=\lambda^{4} \int d t_{x} A(\vec{r}) A\left(r+\left(t_{x}, 0\right)\right)\left[\frac{\sin \left(2 \pi \alpha\left(2 r_{x} t_{x}+t_{x}^{2}\right)\right)}{2 \pi \alpha\left(2 r_{x} t_{x}+t_{x}^{2}\right)}\right]\left[\frac{\sin \left(\phi(\vec{r})-\phi\left(\vec{r}+\left(t_{x}, 0\right)\right)\right)}{\pi t_{x}}\right],
$$

where $\alpha$ is the peak-to-valley defocus error (in units of waves) at the edge of the range gate. Secondly, for an extended NGS with a symmetric intensity profile $W(\vec{\theta})$, the formula becomes

$$
g_{x}(\vec{r}, \phi)=\lambda^{4} \int d t_{x} A(\vec{r}) A\left(r+\left(t_{x}, 0\right)\right) \widehat{W}\left(\left(t_{x}, 0\right) / \lambda\right)\left[\frac{\sin \left(\phi(\vec{r})-\phi\left(\vec{r}+\left(t_{x}, 0\right)\right)\right)}{\pi t_{x}}\right] .
$$

Sample values of the extended object filter function $\widehat{W}$ include:

$$
\widehat{W}(t / \lambda)=\left\{\begin{array}{ll}
J_{0}(\alpha \pi t / 2) & \text { Circular modulation of diameter } D_{m}=\alpha(\lambda / D), \\
\frac{4 J_{1}(\alpha \pi t / 2)}{\alpha \pi t} & \text { Uniform disk of diameter } D_{o}=\alpha(\lambda / D) .
\end{array} .\right.
$$

It should be noted that Eq. (9) depends upon the spatial symmetry of the intensity profile $W$. An asymmetrical source introduces additional terms in the formula, and consequently a nonzero measurement bias with a null wavefront. This effect has been confirmed (qualitatively) in simple simulations of toy extended objects, but has not yet been studied quantitatively.

\subsection{PWFS Case}

The derivation of a measurement formula for the PWFS with an extended source is analogous. Combining the LGS and NGS cases together, the result is the expression:

$$
\begin{aligned}
& g_{x}(\vec{r}, \phi)=\frac{\lambda^{4}}{2} \int d t_{x} A(\vec{r}) A\left(r+\left(t_{x}, 0\right)\right) \widehat{W}\left(\left(t_{x}, 0\right) / \lambda\right)\left[\frac{\sin \left(\alpha\left(2 r_{x} t_{x}+t_{x}^{2}\right)\right)}{\alpha\left(2 r_{x} t_{x}+t_{x}^{2}\right)}\right]\left[\frac{\sin \left(\phi(\vec{r})-\phi\left(\vec{r}+\left(t_{x}, 0\right)\right)\right)}{\pi t_{x}}\right] \\
& \quad-\frac{\lambda^{4}}{2} \iint \frac{d t_{y}}{\pi t_{y}} \frac{d t_{y}^{\prime}}{\pi t_{y}^{\prime}} \int d t_{x} A\left(\vec{r}+\left(0, t_{y}^{\prime}\right)\right) A(\vec{r}+\vec{t}) \\
& \times \widehat{W}\left(\frac{\left|\vec{t}-\left(0, t_{y}^{\prime}\right)\right|}{\lambda}\right)\left[\frac{\sin \left(2 \pi \alpha\left(\left|\vec{r}+\left(0, t_{y}^{\prime}\right)\right|^{2}-|\vec{r}+\vec{t}|^{2}\right)\right)}{2 \pi \alpha\left(\left|\vec{r}+\left(0, t_{y}^{\prime}\right)\right|^{2}-|\vec{r}+\vec{t}|^{2}\right)}\right]\left[\frac{\sin \left(\phi\left(\vec{r}+\left(0, t_{y}^{\prime}\right)\right)-\phi(\vec{r}+\vec{t})\right)}{\pi t_{x}}\right]
\end{aligned}
$$

\section{INFLUENCE MATRIX CALCULATIONS}

The RWFS and PWFS measurement models described above have been used to compute Zernike mode influence matrices. Point source results for Z7 on a $65 \times 65$ point pupil are illustrated in Fig. 2 below. The RWFS and PWFS measurements with no modulation are similar to the analytically computed wavefront gradients (and have also been double-checked again Fourier optics calculations). The reduction in sensitivity due to source modulation is evident from the figures, and is roughly a factor of two in this case. Similar results have been obtained for extended (disk) source and elongated guide stars. 


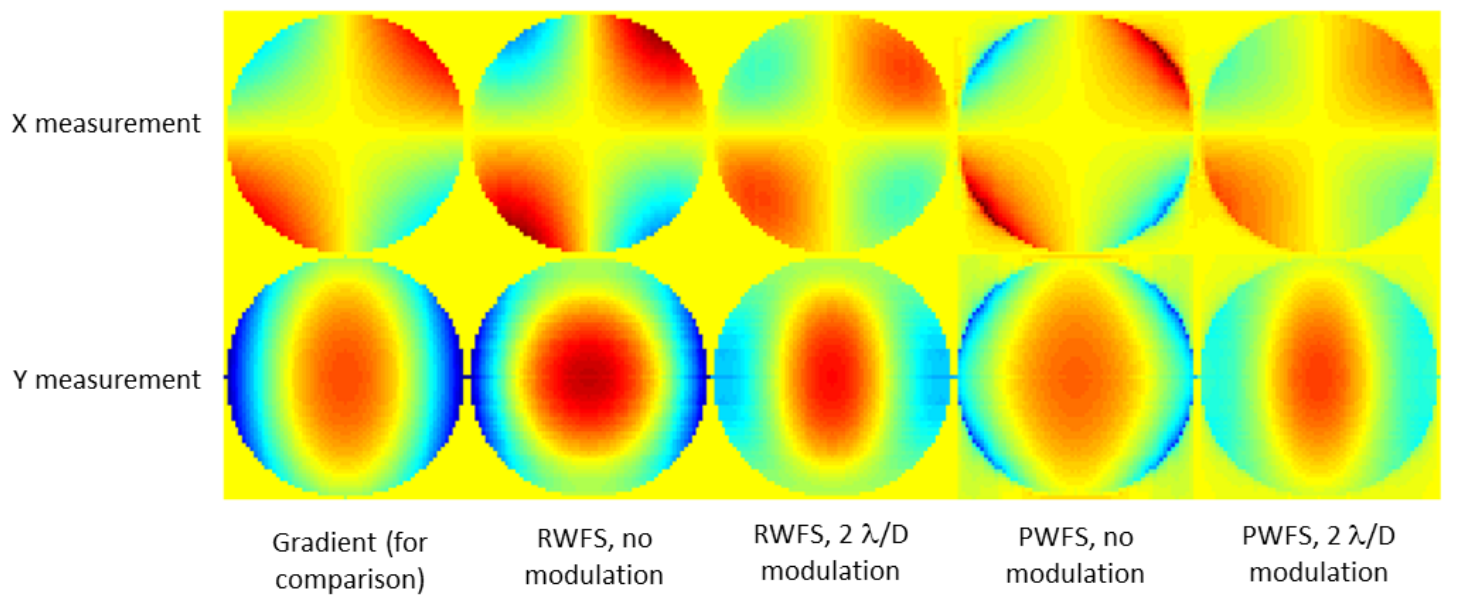

Figure 2. RWFS and PWFS Measurements of Y-Coma. The $x$ - and $y$-measurements with and without modulation are plotted with a common color scale to illustrate the reduction in sensitivity due to modulation.

\section{WAVEFRONT RECONSTRUCTION ERROR DUE TO NOISE FOR THE ROOF WFS}

The Zernike mode influence matrices computed as above can be used to assess the wavefront estimation error due to measurement noise for a nominal unweighted, least squares wavefront reconstruction algorithm. The following plots in Fig. 3 below illustrate the mean-square estimation error due noise as a function of the size of the extended source and the number of Zernike modes estimated, normalized with respect to the error in estimating a single tilt mode with a point source.

In general, these results illustrate that the effect of extended objects on the reconstruction error due to noise is relatively modest for objects no larger than $\lambda / D$, or focus range gates smaller than one wave peak-to-valley. For larger objects or range gates, the reconstruction error in the low-order modes appears to increase roughly with the square of the blurring.

\section{WAVEFRONT RECONSTRUCTION ERROR DUE TO NOISE FOR THE PYRAMID WFS}

Computing influence matrices for the PWFS is computationally intensive because of the triple integrals to be evaluated. The sample preliminary results illustrated in Fig. 4, computed with $65 \times 65$ point pupils, indicate that the trends in the reconstruction error due to noise are at least qualitatively similar to the RWFS, although not identical.

\section{SUMMARY}

In this paper, we have generalized the standard measurement formulas for the roof- and pyramid wavefront sensors (RWFS and PWFS) to the case of extended sources. The extended source introduces a filter function into the measurement formula, which has the effect of reducing sensitivity and consequently increasing the wavefront reconstruction error due to noise. We have quantified this impact for three sample cases of extended sources: An idealized laser guide star with a top-hat sodium layer (or backscatter) profile, a disk source, and a point source with circular modulation. In each case, the effect of the extended object is relatively modest until the size of the object exceeds a diameter of $\lambda / D$, or one wave peak-to-valley defocus in the case of an LGS. After that, the mean-square wavefront reconstruction error in the low order modes appears to increase roughly with the square of the size of the source. This result is qualitatively consistent with the intuition that the sensitivity of the PWFS [RWFS] is dependent upon obtaining a well-focused spot at the tip of the pyramid [roof]. 

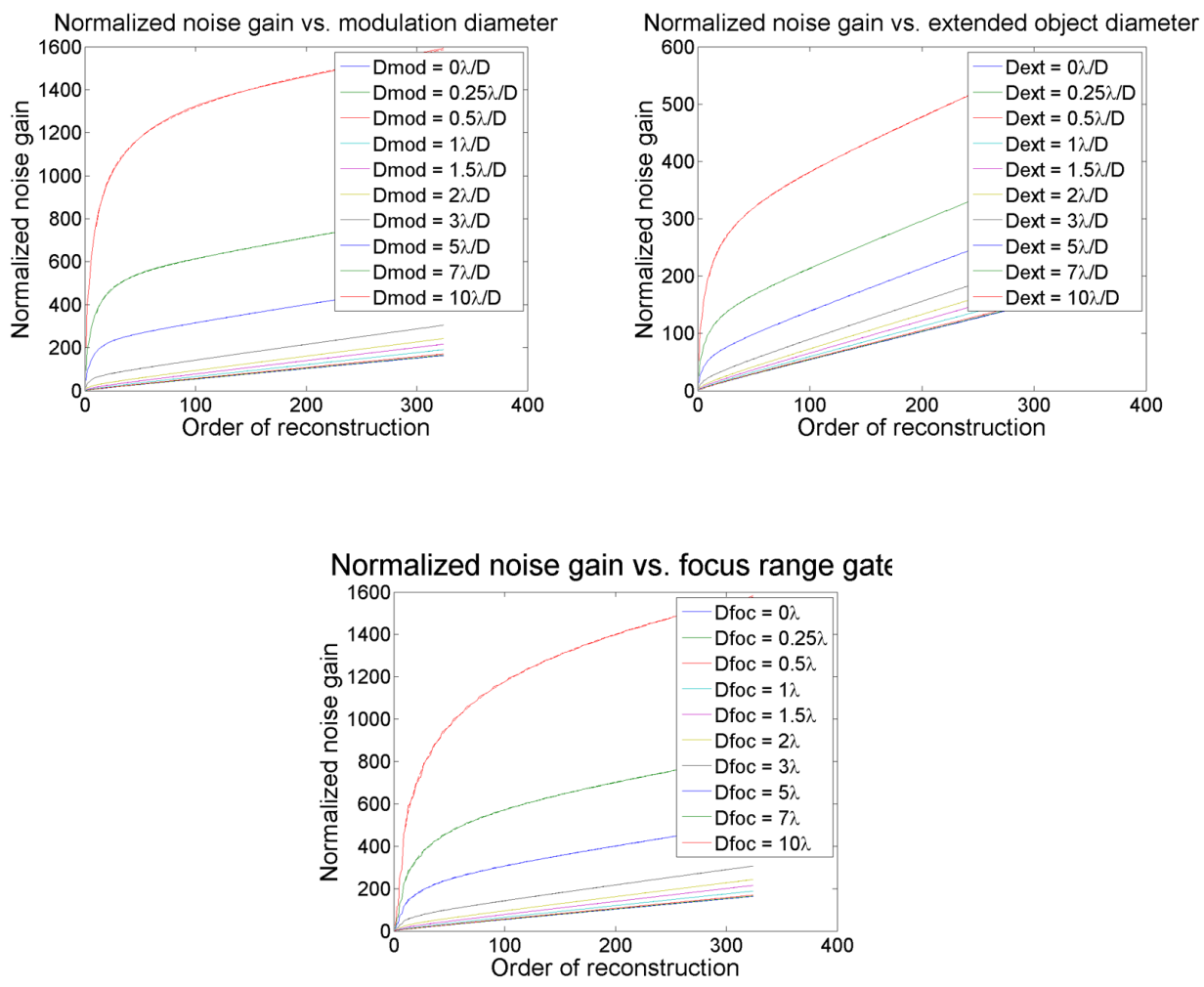

Figure 3. Relative wavefront reconstructor noise gain vs. extended source size and the number of Zernike modes reconstructed for the RWFS.

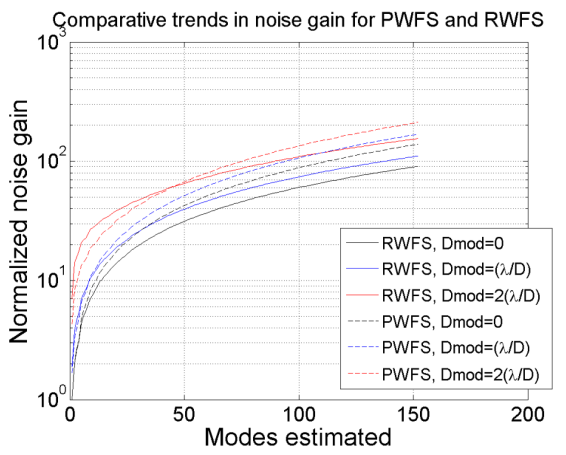

Figure 4. Comparative trends in reconstructor noise gain for the RWFS and PWFS.

\section{ACKNOWLEDGMENTS}

The TMT Project gratefully acknowledges the support of the TMT collaborating institutions. They are the California Institute of Technology, the University of California, the National Astronomical Observatory of Japan, the National Astronomical Observatories of China and their consortium partners, the Department of Science and Technology of India and their supported institutes, and the National Research Council of Canada. This work was supported as well by the Gordon and Betty Moore Foundation, the Canada Foundation for Innovation, the Ontario Ministry of Research and Innovation, the Natural Sciences and Engineering Research Council of Canada, the British Columbia Knowledge Development Fund, the Association of Canadian Universities for Research in Astronomy (ACURA), the Association of Universities for Research in Astronomy (AURA), the U.S. National Science Foundation, the National Institutes of Natural Sciences of Japan, and the Department of Atomic Energy of India. 


\section{REFERENCES}

1. Roberto Ragazzoni, Pupil plane wavefront sensing with an oscillating prism. Journal of Modern Optics, 43(2):289-93, 1996.

2. Visa Korkiakoskia and Christophe Verinaud, Extreme adaptive optics simulations for EPICS. In 1st AO4ELT Conference, http://dx.doi.org/10.1051/ao4elt/201003007, 2010.

3. Anna Burvall et al, Linearity of the pyramid wavefront sensor, Optics Express 14(25):11925-34, 2006. https://doi.org/10.1364/OE.14.011925

\section{APPENDIX A. RWFS AND PWFS MEASUREMENT MODELS WITH A POINT SOURCE}

The usual Fourier optics model for the RWFS and PWFS measurements are given by the two equations

$$
\begin{aligned}
g_{\{x, y\}}(\vec{r}, \phi) & =\sum_{i} w_{\{x, y\}, i} I\left(\vec{r}, \phi, m_{i}\right) \\
I(\vec{r}, \phi, m) & =\left|\int d \vec{\kappa} m(\vec{\kappa}) \exp (k \vec{r} \cdot \vec{\kappa}) \int d \vec{s} A(\vec{s}) \exp [i \phi(\vec{s})] \exp (-k \vec{s} \cdot \vec{\kappa})\right|^{2},
\end{aligned}
$$

where the variables are defined in Section 2 above. Eq. (13) may be rewritten in the form

$$
\begin{aligned}
I(\vec{r}, \phi, m)= & \iint d \vec{\kappa} d \vec{\kappa}^{\prime} m(\vec{\kappa}) m\left(\vec{\kappa}^{\prime}\right) \exp \left[i k \vec{r} \cdot\left(\vec{\kappa}-\vec{\kappa}^{\prime}\right)\right] \\
& \times \iint d \vec{s} d \vec{s}^{\prime} A(\vec{s}) A\left(\vec{s}^{\prime}\right) \exp \left[-i k\left(\vec{s} \cdot \vec{\kappa}-\vec{s}^{\prime} \cdot \vec{\kappa}^{\prime}\right)\right] \exp \left[i\left(\phi(\vec{s})-\phi\left(\vec{s}^{\prime}\right)\right)\right],
\end{aligned}
$$

and substituting this expression into Eq. (12) and commuting the order of the integrations and summation yields the result

$$
g_{\{x, y\}}(\vec{r}, \phi)=\iint d \vec{s} d \vec{s}^{\prime} A(\vec{s}) A\left(\vec{s}^{\prime}\right) \exp \left[i\left(\phi(\vec{s})-\phi\left(\vec{s}^{\prime}\right)\right)\right] \sum_{i} w_{\{x, y\}, i} \widehat{m}_{i}[(\vec{s}-\vec{r}) / \lambda] \widehat{m}_{i}^{*}\left[\left(\vec{s}^{\prime}-\vec{r}\right) / \lambda\right] .
$$

The RWFS and PWFS cases are evaluated further below in the following two subsections.

\section{A.1 RWFS Case}

For the RWFS, the pair of spatial filters $m_{1}$ and $m_{2}$ are given by

$$
\begin{aligned}
& m_{1}(\vec{p})= \begin{cases}1 & 0 \leq p_{1} \leq P \\
0 & \text { otherwise }\end{cases} \\
& m_{2}(\vec{p})=m_{1}(-\vec{p}),
\end{aligned}
$$

where $P$ is the half-width of a finite field stop in the plane of the roof. By introducing this field stop, the Fourier transforms of the spatial filters can be evaluated as

$$
\begin{aligned}
\widehat{m_{1}}(\vec{\nu}) & =\delta\left(\nu_{2}\right) \exp \left(-\pi i \nu_{1} P\right) \frac{\sin \left(\pi \nu_{1} P\right)}{\pi \nu_{1}} \\
\widehat{m_{2}}(\vec{\nu}) & ={\widehat{m_{1}}}^{*}(\vec{\nu}),
\end{aligned}
$$

where $\delta$ denotes the usual Dirac delta function. Taking a limit as $P$ approaches infinity will then yield the desired result.

Using Eq. (18) and Eq. (19), the summation appearing at the end of Eq. (15) becomes

$$
\begin{aligned}
\sum_{i} w_{x, i} \widehat{m}_{i}[(\vec{s}-\vec{r}) / \lambda] \widehat{m}_{i}^{*}\left[\left(\vec{s}^{\prime}-\vec{r}\right) / \lambda\right]= & \delta\left[\left(s_{2}-r_{2}\right) / \lambda\right] \delta\left[\left(s_{2}^{\prime}-r_{2}\right) / \lambda\right] 2 i \sin \left[\pi\left(s_{1}^{\prime}-s_{1}\right) P / \lambda\right] \\
& \times \frac{\sin \left[\pi\left(s_{1}-r_{1}\right) P / \lambda\right]}{\pi\left(s_{1}-r_{1}\right) / \lambda} \frac{\sin \left[\pi\left(s_{1}^{\prime}-r_{1}\right) P / \lambda\right]}{\pi\left(s_{1}^{\prime}-r_{1}\right) / \lambda}
\end{aligned}
$$


The two delta functions can be used to evaluate the integrals with respect to $s_{2}$ and $s_{2}^{\prime}$ in Eq. (15), yielding the result

$$
\begin{aligned}
g_{x}(\vec{r}, \phi)=\quad \lambda^{2} & \iint d s_{1} d s_{1}^{\prime} A\left(s_{1}, r_{2}\right) A\left(s_{1}^{\prime}, r_{2}\right) \exp \left[-i\left(\phi\left(s_{1}, r_{2}\right)-\phi\left(s_{1}^{\prime}, r_{2}\right)\right)\right] \\
& \times 2 i \sin \left[\pi\left(s_{1}^{\prime}-s_{1}\right) P / \lambda\right] \frac{\sin \left[\pi P\left(s_{1}-r_{1}\right) / \lambda\right]}{\pi\left(s_{1}-r_{1}\right) / \lambda} \frac{\sin \left[\pi P\left(s_{1}^{\prime}-r_{1}\right) / \lambda\right]}{\pi\left(s_{1}^{\prime}-r_{1}\right) / \lambda}
\end{aligned}
$$

By applying the changes of variable $t_{1}=s_{1}-r_{1}$ and $t_{1}^{\prime}=s_{1}^{\prime}-r_{1}$, this now becomes

$$
\begin{aligned}
g_{x}(\vec{r}, \phi)=\lambda^{2} & \iint d t_{1} d t_{1}^{\prime} A\left(t_{1}+r_{1}, r_{2}\right) A\left(t_{1}^{\prime}+r_{1}, r_{2}\right) \exp \left[-i\left(\phi\left(t_{1}+r_{1}, r_{2}\right)-\phi\left(t_{1}^{\prime}+r_{1}, r_{2}\right)\right)\right] \\
& \times 2 i\left\{\frac{\sin \left(k P t_{1}\right)}{k t_{1}} \frac{1-\cos \left(k P t_{1}^{\prime}\right)}{k t_{1}^{\prime}}-\frac{1-\cos \left(k P t_{1}\right)}{k t_{1}} \frac{\sin \left(k P t_{1}^{\prime}\right)}{k t_{1}^{\prime}} .\right\}
\end{aligned}
$$

Finally, we take the limit of this expression as $P$ approaches infinity. The limits of the trigonometric terms within braces take the form

$$
\begin{gathered}
\lim _{P \rightarrow \infty} \frac{1-\cos (k P x)}{k x}=\frac{1}{k x} \\
\lim _{P \rightarrow \infty} \frac{\sin (k P x)}{k x}=\frac{\lambda}{2} \delta(x) .
\end{gathered}
$$

Note that these limits are not pointwise, but instead in the sense of a distribution, i.e.

$$
\lim _{P \rightarrow \infty} \int d x\left[\frac{1-\cos (k P x)}{k x}\right] f(x)=\int d x\left[\frac{1}{k x}\right] f(x),
$$

for suitably smooth functions $f(x)$, and similarly for $\sin (k P x) /(k x)$.

Substituting Eq.'s (23) and (24) back into Eq. (22) and evaluating integrals where possible using the delta functions now yields the desired formula for the RWFS measurements, namely

$$
g_{x}(\vec{r}, \phi)=\lambda^{4} \int d t_{1} A(\vec{r}) A\left(r_{1}+t_{1}, r_{2}\right) \sin \left[\phi(\vec{r})-\phi\left(r_{1}+t_{1}, r_{2}\right)\right] /\left(\pi t_{1}\right)
$$

\section{A.2 PWFS Case}

For the PWFS, there are four spatial filtering functions $m_{ \pm, \pm}$and 4 quad cell weights $w_{ \pm, \pm}$, which are defined by the equations:

$$
\begin{aligned}
m_{ \pm, \pm}(\vec{\theta}) & = \begin{cases}1 & \text { if }\left|\theta_{1} \pm P / 2\right| \leq P / 2 \\
& \text { and }\left|\theta_{2} \pm P / 2\right| \leq P / 2 \\
0 & \text { otherwise }\end{cases} \\
w_{-,-} & =w_{-,+}=1 \\
w_{+,-} & =w_{+,+}=-1
\end{aligned}
$$

The Fourier transforms of the spatial filters now become

$$
\widehat{m}_{ \pm, \pm}(\vec{\kappa})=\exp \left[-i \pi P\left( \pm \kappa_{1} \pm \kappa_{2}\right)\right] \frac{\sin \left(\pi P \kappa_{1}\right)}{\pi \kappa_{1}} \frac{\sin \left(\pi P \kappa_{2}\right)}{\pi \kappa_{2}} .
$$

Making the substitutions $\vec{t}=\vec{s}-\vec{r}$ and $\vec{t}=\vec{s}-\vec{r}$ the weighted sum of the transforms appearing at the end of Eq. (15) may be evaluated as

$$
\begin{aligned}
\sum_{j=1}^{4} m_{j} \widehat{w}_{j}(\vec{t} / \lambda) \widehat{w}_{j}^{*}\left(\overrightarrow{t^{\prime}} / \lambda\right) & =4 i\left[\frac{1-\cos \left(k P t_{1}\right)}{k t_{1}} \frac{\sin \left(k P t_{1}^{\prime}\right)}{k t_{1}^{\prime}}-\frac{\sin \left(k P t_{1}\right)}{k t_{1}} \frac{1-\cos \left(k P t_{1}^{\prime}\right)}{k t_{1}^{\prime}}\right] \\
& =\times\left[\frac{\sin \left(k P t_{2}\right)}{k t_{2}} \frac{\sin \left(k P t_{2}^{\prime}\right)}{k t_{2}^{\prime}}-\frac{1-\cos \left(k P t_{2}\right)}{k t_{2}} \frac{1-\cos \left(k P t_{2}^{\prime}\right)}{k t_{2}^{\prime}}\right]
\end{aligned}
$$


and taking the limit of this expression as $P$ increases to infinity yields the result

$$
\lim _{P \rightarrow \infty} \sum_{j=1}^{4} m_{j} \widehat{w}_{j}(\vec{t} / \lambda) \widehat{w}_{j}^{*}(\vec{t} / \lambda)=\frac{i \lambda^{4}}{4}\left[\frac{1}{\pi t_{1}} \delta\left(t_{1}^{\prime}\right)-\delta\left(t_{1}\right) \frac{1}{\pi t_{1}^{\prime}}\right]\left[\delta\left(t_{2}\right) \delta\left(t_{2}^{\prime}\right)-\frac{1}{\pi t_{2}} \frac{1}{\pi t_{2}^{\prime}}\right] .
$$

One again, this limit should be interpreted in the sense of a distribution. Eq. (11) for the PWFS measurement can now be obtained by substituting this formula in Eq. (15), and evaluating the integrals where possible using the delta functions.

\section{APPENDIX B. RWFS AND PWFS MEASUREMENT MODELS WITH EXTENDED SOURCES}

For a spatially extended guide star, the wavefront from each point in the object is adjusted by tip/tilt and focus terms:

$$
\begin{aligned}
\phi_{o}(\vec{r}) & =\phi_{o}(\vec{r} ; \vec{\theta}, f) \\
& =\frac{2 \pi \vec{\theta}}{(\lambda / D)} \cdot\left(\frac{\vec{r}}{D}\right)+2 \pi f\left|\frac{2 \vec{r}}{D}\right|^{2} .
\end{aligned}
$$

Here $\vec{\theta}$ is the angular coordinate of the point and (in the case of an LGS) $f$ is the range coordinate given in units of peak-to-valley waves of defocus across the telescope aperture. The following two subsections assess the impact of these terms on the RWFS and PWFS measurement equations for the two sample cases of a "pure" LGS $(\vec{\theta} \equiv 0)$ and a symmetric extended object.

\section{B.1 LGS case}

With $\vec{\theta}=0$, the phase difference term appearing in the RWFS and PWFS measurement equations takes the form

$$
\begin{aligned}
& \sin \left[\phi(\vec{r}+\vec{s})-\phi\left(\vec{r}+\vec{s}^{\prime}\right)+\phi_{o}(\vec{r}+\vec{s})-\phi_{o}\left(\vec{r}+\vec{s}^{\prime}\right)\right]= \\
& \quad \sin \left[\phi(\vec{r}+\vec{s})-\phi\left(\vec{r}+\vec{s}^{\prime}\right)\right] \cos \left[(2 \pi f)\left(\left|\frac{2(\vec{r}+\vec{s})}{D}\right|^{2}-\left|\frac{2\left(\vec{r}+\vec{s}^{\prime}\right)}{D}\right|^{2}\right)\right] \\
& \quad+\cos \left[\phi(\vec{r}+\vec{s})-\phi\left(\vec{r}+\vec{s}^{\prime}\right)\right] \sin \left[(2 \pi f)\left(\left|\frac{2(\vec{r}+\vec{s})}{D}\right|^{2}-\left|\frac{2\left(\vec{r}+\vec{s}^{\prime}\right)}{D}\right|^{2}\right)\right] .
\end{aligned}
$$

Integrating over $f$ for the case of a symmetric, top-hat range gate yields

$$
\frac{1}{2 \alpha} \int_{-\alpha}^{\alpha} d f p(f) \sin \left[\phi(\vec{r}+\vec{s})-\phi\left(\vec{r}+\vec{s}^{\prime}\right) d z\right]=\sin \left[\phi(\vec{r}+\vec{s})-\phi\left(\vec{r}+\vec{s}^{\prime}\right)\right] \frac{\sin \left[2 \pi \alpha\left(\left|\frac{2(\vec{r}+\vec{s})}{D}\right|^{2}-\left|\frac{2\left(\vec{r}+\vec{s}^{\prime}\right)}{D}\right|^{2}\right)\right]}{2 \pi \alpha\left(\left|\frac{2(\vec{r}+\vec{s})}{D}\right|^{2}-\left|\frac{2\left(\vec{r}+\vec{s}^{\prime}\right)}{D}\right|^{2}\right)},
$$

where the variable $\alpha$ is the half-width of the range gate. Substituting the appropriate values for $\vec{s}$ and $\overrightarrow{s^{\prime}}$ now yields the range gate terms appearing in Eq.'s (8) and (11).

\section{B.2 Extended Object Case}

In the alternative case where $f=0$, the phase difference term becomes instead

$$
\begin{aligned}
\sin \left[\phi(\vec{r}+\vec{s})-\phi\left(\vec{r}+\vec{s}^{\prime}\right)+\phi_{o}(\vec{r}+\vec{s})-\phi_{o}\left(\vec{r}+\vec{s}^{\prime}\right)\right]= & \sin \left[\phi(\vec{r}+\vec{s})-\phi\left(\vec{r}+\vec{s}^{\prime}\right)\right] \cos \left[\frac{2 \pi \vec{\theta}}{(\lambda / D)} \cdot \frac{\left(\vec{s}-\vec{s}^{\prime}\right)}{D}\right] \\
& +\cos \left[\phi(\vec{r}+\vec{s})-\phi\left(\vec{r}+\vec{s}^{\prime}\right)\right] \sin \left[\frac{2 \pi \vec{\theta}}{(\lambda / D)} \cdot \frac{\left(\vec{s}-\vec{s}^{\prime}\right)}{D}\right]
\end{aligned}
$$


If the extended object $W(\vec{\theta})$ is symmetric in $\vec{\theta}$, integrating over the object intensity profile yields

$$
\begin{aligned}
\int d \vec{\theta} W(\vec{\theta}) \sin \left[\phi(\vec{r}+\vec{s})-\phi\left(\vec{r}+\vec{s}^{\prime}\right)\right] & =\sin \left[\phi(\vec{r}+\vec{s})-\phi\left(\vec{r}+\vec{s}^{\prime}\right)\right] \int d \vec{\theta} W(\vec{\theta}) \cos \left[\frac{2 \pi \vec{\theta}}{(\lambda / D)} \cdot \frac{\left(\vec{s}-\vec{s}^{\prime}\right)}{D}\right] \\
& =\sin \left[\phi(\vec{r}+\vec{s})-\phi\left(\vec{r}+\vec{s}^{\prime}\right)\right] \widehat{W}\left[\left(\vec{s}-\vec{s}^{\prime}\right) / \lambda\right]
\end{aligned}
$$

Similarly to the LGS range gate case described above, this yields the extended object term appearing in Eq.'s (8) and (11) via substituting the appropriate values for $\vec{s}$ and $\vec{s}$.

Note that the second term in Eq. (37) yields a value of zero when integrated because sin is an odd function and the intensity profile $W$ is assumed to be symmetric. If $W$ is not symmetric, the second term in Eq. (37) will contribute to the value of the integral. In particular, it will introduce a bias in the PWFS or RWFS measurement when $\phi \equiv 0$. This result has been verified qualitatively in simulations of toy extended objects, but futher study is required to assess the significance of the effect. 